\title{
Thomas Bernhards „Ein Kind“: Erste Ein- und Aussichten zu Wortschatz und Wort(sequenz)verwendung
}

\author{
Manfred B. Sellner \\ Universität Salzburg \\ $\&$ \\ Academiae Corpora (ÖAW) \\ manfred.sellner@sbg.ac.at
}

\begin{abstract}
Der Aufsatz beschäftigt sich mit quantitativen Aspekten des autobiografischen Romans „Ein Kind“ von Thomas Bernhard. Das Ziel dieses deskriptiv konzipierten Pilotprojekts besteht darin, mit konventionellen Methoden der computergestützten Korpuslinguistik präzise Aussagen zu den quantitativen Eigenschaften von ausgewählten Lexemen (Unigrammen) und Lexemsequenzen (Bigrammen, Trigrammen, etc.) des Romans zu formulieren. Die Ergebnisse sollen als mögliche Grundlage und Referenz für Analysen mit größerer Datenbasis und erweiterter Methode sowie zum Vergleich der Sprachverwendung in literarischen Werken dienen. Das Ziel des Forschungsprojektes ist die Explikation von Aspekten der ,literarischen Sprachverwendung“.
\end{abstract}

\section{Einleitung}

Thomas Bernhard gilt als einer der profiliertesten und zugleich provokantesten österreichischen Autoren der 2. Hälfte des 20. Jahrhunderts. Er hinterlässt ein voluminöses Oeuvre, zu dem auch fünf autobiographische Romane zählen. ${ }^{1}$ Aus dieser Sequenz von Ich-Romanen wird hier der 1982 erschienene Band „Ein Kind“ zur Analyse verwendet. ${ }^{2}$

Das Ziel dieser Arbeit besteht in dem Versuch, einige allgemeine Erkenntnisse über die lexikalische und syntaktisch-sequentielle Sprachverwendung von Thomas Bernhards Roman zu gewinnen, welche mit gleichen, oder vielleicht auch modifizierten Methoden der Korpuslinguistik, und unter Verwendung einer größeren Datenbasis generelle Aussagen nicht nur zur autorenspezifischen Stilistik von Thomas Bernhard treffen können, sondern auch zur generellen Charakterisierung von „literarischer Sprache“ führen können. Die Untersuchung beginnt mit der Feststellung der Quantitäten und der Distribution von einzelnen Unigrammen (,Einzellexemen“), wobei exemplarisch das quantitative Auftreten von Nennungen der Verwandtschaft von Thomas Bernhard analysiert wird, welche im Leben, und dann konsequenterweise als lexikalisches Spiegelbild im Roman „Ein Kind“ als wichtige Bezugspersonen angesehen werden können. Die Analyse dieses Wortschatzteils von „Bigrammen“ führt dann zur Analyse und Illustration der weiteren Mehrfachverwendung von Lexemen und Lexemsequenzen, welche mit der Methode der Herauslösung und Quantifizierung von Multigrammen („Trigrammen“, „Quagrammen“, und schließlich "Quingrammen“) durchgeführt wird. Diese Methodik soll somit als Schritt zur Beantwortung der Frage „Gibt es bei Thomas Bernhard eine große lexikalische und phrasale Diversität und somit große linguistische Kreativität?", dienen.

Die Methodik der Korpusanalyse wird hier größtenteils nach der „Tidy-Methode“, welche im Rahmen der Domäne der „R-Statistikumgebung“ entwickelt wurde, durchgeführt. ${ }^{3}$ Diese wurde in

\footnotetext{
${ }^{1}$ Als autobiografische Romane von Thomas Bernhard gelten die folgenden 5 Werke: „Der Atem“, „Der Keller“, „Die Kälte“ und „Die Ursache“ und „Ein Kind“.

"Ich möchte mich bei der ÖAW/Academiae Corpora für die Bereitstellung der Digitalisate bedanken. Ohne die Digitalisate wäre diese Arbeit nicht möglich gewesen. Siehe https://thomasbernhard.at/index.php?id=226\&L=0, wie auch Huber, Martin \& Manfred Mittermayer (Hrsg.) 2004 und die dort genannten Arbeiten zur literaturwissenschaftlichen Rezeption von Thomas Bernhard.

${ }^{3}$ Siehe: The R Foundation for Statistical Computing.
} 
Silge \& Robinson (2017), wie auch in Wickham \& Grolemund $\left(2017^{2}\right)$, vorgestellt und illustriert. Für diese konkrete Analyse des Bernhard-Romans wurden hier gemäß der "Tidy-Methode“ verschiedene Funktionen der Kernpakete von R wie auch von "tidyverse", "tidytext" und "dplyr" zur korpuslinguistischen Analyse verwendet. Ein wichtiges Charakteristikum für diese Analysemethode ist, dass der Output der Funktionen ein Datenformat ausgibt, das als „Tibble“ bezeichnet wird. Es wird als "table with one token per row" definiert und kann dann als Input für die weitere statistische Modellierung der Daten herangezogen werden. ${ }^{4}$ Zum Begriff „Wort“, „Wortform“, „Token“, aber auch „Wortfolge“ und „Wortsequenz“, etc. ist hier zu erwähnen, dass es sich jeweils um eine orthografische Sequenz bzw. als eine orthografische Sequenzfolge betrachtet wird, die mittels computerlinguistischen Programmanweisungen in Textteile (auch „Token“ genannt) getrennt wird. Die Verwendung des Begriffs „Wort“ und „Wortfolge“ soll neutral gegenüber den Unterscheidungen von „morphologischem“, „phonologischem“ und „syntaktischem“ Wort verstanden werden. Wort, wie auch Token, etc. werden, wie in der computergestützten Korpuslinguistik üblich, einfach als Zeichenfolge zwischen zwei Leerräumen charakterisiert. ${ }^{5}$ Diese Interpretation von „Wort“ bedingt jedoch beispielsweise, dass die Wortformen „Großvater" und „Großvaters“ als zwei verschiedene Tokens und somit als 2 Wörter analysiert und gezählt werden.

\section{2 „Ich“ und „meine Verwandten“: Analysen von Unigrammen}

Der erste Analyseschritt einer korpuslinguistischen Analyse besteht im Einlesen eines Textes in den PC, um einen Text für den Computer „les- und interpretierbar“ zu machen. Dies erfolgt hier mit der Statistiksoftware R und seiner Kernfunktion "scan”. Der nächste Schritt im „Workflow“ ist die „Tokenisierung“ des Textes, welche zur Trennung des Textes in für den Computer interpretierbare „Wörter“ dient. Die Ausführung dieser Arbeitsschritte zeigt in Bezug auf den Roman „Ein Kind“, dass der Output der diesbezüglichen Funktionen - wie in Tabelle (1) vorgestellt - aus 31.671 Zeilen ("rows") besteht und somit aus 31.671 "words" („Wörtern/Wortfolgen/Buchstabenfolgen“) besteht. Standardmäßig besteht der Output aus 10 Zeilen mit je einem Wort und hier daher um die ersten 10 „Wörter“ des Romanbeginns. ${ }^{6}$ Die Tabelle zeigt auch, dass die Elemente als Spalten ausgegeben werden und die für uns wichtige Spaltenvariable vom R-Programm als "word" ausgewiesen wird.

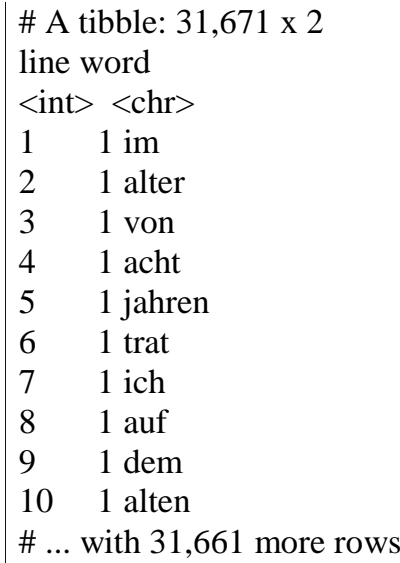

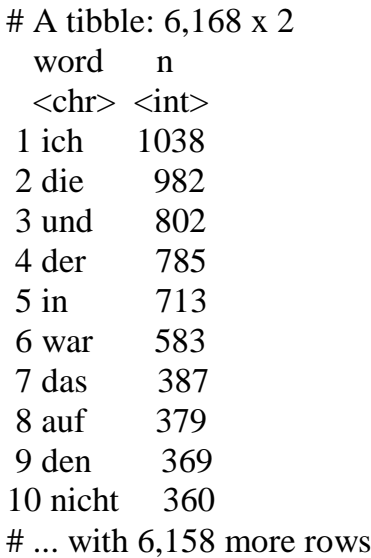

Im Zusammenhang mit dem Output der "unnest_tokens"-Funktion ist zu erwähnen, dass die Spaltenelemente von Tabelle (1), die gemäß der deutschen Orthografiekonvention und so auch im Roman von Thomas Bernhard, normalerweise mit Großbuchstaben geschrieben werden (z. B. „Alter“, „Jahre“), durch die Anwendung der „Tokenisierungsfunktion“ in Kleinbuchstaben konvertiert werden.

\footnotetext{
${ }^{4}$ Vgl. Silge \& Robinson (2017:1).

5 Zur auch für die Korpuslinguistik relevanten Diskussion um den Begriff „Wort” und den Begriffen „Token“ und „Type” siehe Perkuhn et. al. 2012:26-28.

${ }^{6}$ Vgl. in diesem Zusammenhang Baayen (2008:223): "We will refer to the number of unique words as the number of types, and to the number of words regardless of their identity as the number of tokens."
} 
Darüber hinaus werden auch die Satzzeichen, welche im Deutschen teilweise die Unterscheidung von Großbuchstaben und Kleinbuchstaben bedingen, wie auch die Orthografieelemente, die Satzteile markieren, getilgt. Dadurch entsteht ein „Tibble“, das ausschließlich aus Kleinbuchstaben besteht, keine Satzzeichen aufweist, und wie Zahlen einer Tabelle vom R-Programm verarbeitet werden kann. Wie schon erwähnt, werden standardmäßig die ersten 10 Zeilen eines „Tibbles“ vom Programm ausgegeben. Um eine größere Menge von Elementen anzuzeigen, muss dies mit einer speziellen Funktion spezifiziert werden (z. B. „n=100“).

Im nächsten Arbeitsschritt wird die Häufigkeit der unterschiedlichen „Token“, also jeder einzelnen Wortform des Textes mit den Funktionen: "count" und "sort" ermittelt. Das Ergebnis der diesbezüglichen Analyse ist die Information von Tabelle (2) oben. Im Detail zeigt uns die Tabelle, dass der Analysetext „Ein Kind“ insgesamt 6.168 “types” (=verschiedene „Typen“ bzw. Wortformen) aufweist. Die einzelnen Typen werden im Output sowohl mit ihrer absoluten Häufigkeit im Text, wie auch mit dem Rang im Text, angegeben. An erster und oberster Stelle steht hier die Buchstabenfolge „ich“ und kommt gemäß der Auszählung durch das Programm im Text 1038 Mal vor. Diese Personenreferenz, welche sich auf den Autor als Hauptprotagonist bezieht, nimmt demgemäß den ersten Rang unter den Typen des Romans ein. Die nächste Wortfolge, d. i. „die“, kommt im Text 982 Mal vor und nimmt damit den 2. Rang ein. Als Ergebnisse dieser Analyseschritte können wir zusammenfassend somit in Bezug auf den quantitativen Aspekt des Textes festhalten, dass der Roman 31.671 „Wörter/Tokens“ und 6.168 „Worttypen“ hat. Dies bedeutet, dass der vorliegende Text eine Type/Token-Relation, - welche ein Maß ist, das den Grad der Komplexität eines Wortschatzes angibt-, von $0.195 \%$ hat. Wenn wir aber das Korpus zum Zwecke des Vergleichs mit anderen Texten verwenden, müssen diese „normalisiert“ werden, indem wir beispielsweise die Anzahl der Types mit 100 multiplizieren. Dies ergibt ein Type/Token-Verhältnis von 19,47\%. Das Ergebnis wird dahingehend interpretiert, dass ein höherer Wert auf eine differenzierte Wortwahl anzeigt und ein niedriger auf „ein größeres Maß an Wiederholung und auf eine formelhaftere Sprachverwendung.“ Die hier berechnete, „,normalisierte Maßzahl“ wäre somit ein Vergleichswert zum Vergleich mit anderen Werken des gleichen Genres. ${ }^{7}$

Da es sich beim Roman um eine Autobiografie, also um einen „Ich-Roman“ handelt, in dem der Autor in schriftstellerischer Diktion über seine Kindheit in Salzburg und Bayern berichtet, überrascht es nicht, dass dieses Personalpronomen im Text von Thomas Bernhard eine so prominente Stelle einnimmt. Aus diesem Grunde sollte eine Visualisierung dieser Beobachtung mittels „Wortwolke“ diese Selbstreferenz in den Mittelpunkt stellen und im Vergleich zu den anderen Elementen am größten sein (Grafik 1, unten). Danach sollten „weniger prominente Lexeme“, wie „die“ und „und“, etc. folgen und um ,ich“ platziert sein. Wie wir unschwer erkennen, wird diese Aussage auch vorbehaltlos bestätigt, da das Lexem ,ich“ vom Programm in die Mitte platziert wurde und die anderen Lexeme das Pronomen „ich“ in unterschiedlicher Größe und in unterschiedlicher Entfernung umringen. ${ }^{8}$

Im folgenden Abschnitt soll der Frage nachgegangen werden, ob große Teile der „Typen“ von Unigrammen („Einzelwörtern“) im Roman eine zahlenmäßig ähnliche Ausprägung aufweisen, oder ob nur ein kleiner Teil der Typen oft vorkommt, während der jeweils viel größere Teil nicht in so hoher Frequenz im Text verwendet wurde. Um dafür einen ersten Eindruck zu gewinnen, soll die folgende Übersichtstabelle (3) dienen, welche die ersten 100 „Wortränge“ und deren zahlenmäßiges Vorkommen im Roman darstellt.

Wie Tabellen (1) und (2) andeuteten, die folgende Tabelle (3) jedoch im größeren Ausmaße illustriert, finden wir in Bezug auf die Vorkommenshäufigkeit der „Types/Typen“ des Romans „Ein Kind“" eine große Streuung vor. Dies zeigt die numerische Information von Tabelle (3) deutlich, da wir erkennen können, dass schon die ersten 100 Tokens des Tibble (die „Top-100 Wörter") eine Spannweite von $n=37$ bis zu $n=1038$ aufweisen.

\footnotetext{
${ }^{7}$ Siehe Perkuhn, et. al. 2018, sowie die „Ergänzungen zu Korpuslinguistik“ vom 18. Juni 2012. Die Autoren erwähnen auch, dass die Type/Token-Relation, welche das Verhältnis angibt „mit welcher Rate die Tokens im Korpus zu unterschiedlichen Typen angehören... immer eine Zahl zwischen 0 und $100 \%$ ergibt.“

${ }^{8}$ Grafik (1) ist eine Abbildung von Rang (1-75) von Tabelle (2).
} 


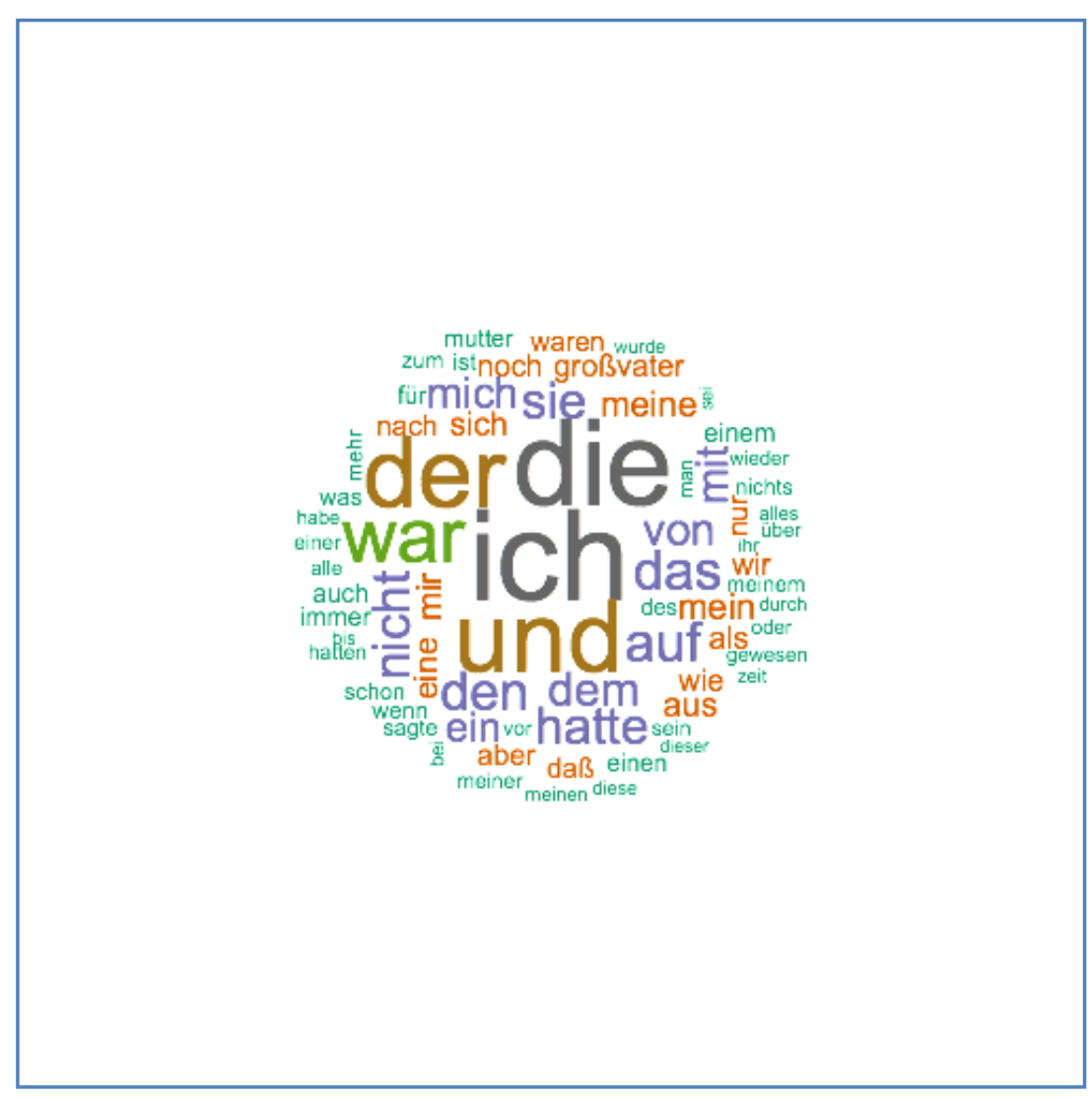

Grafik (1): Wortwolke

Wie aus Tabelle (3) weiters ersichtlich, kommen neben den 4 mit Thomas Bernhard verwandtschaftlich verbundenen und im Roman oft genannten Personen wie „Großvater“ (Rang:29), „Mutter“ (Rang:44), „Vater“ (Rang:90) und „Großmutter“ (Rang:92) nur noch 2 weitere Nomina, das sind „Zeit" und „Schule“ vor. Darüber hinaus finden wir noch einige Auxiliar- und Verbformen wie „war“, „hatte“, „sagte“, „,hatten“, „gewesen”, „habe“, „wurde“ und „musste“ vor. Den übergroßen Anteil an Wortformen in der „Top-100-Liste“ machen jedoch sogenannte „Funktionswörter" aus. Die Vertreter dieser auch „Stopwords“ genannten Wortart werden in Korpusanalysen sehr oft getilgt, weil man annimmt, dass sie keinen Einfluss auf die Semantik und Pragmatik eines Textes haben. Da hier jedoch das Hauptaugenmerk auf eine Explikation der lexikalischen Quantitäten gelegt ist, wird von einer Analyse des Textes ohne Stopwords abgesehen. Im nächsten Abschnitt wollen wir der Frage nachgehen, ob die in Tabelle (3) aufgelistete Streuung von types als Zufallsverteilung angesehen werden muss, oder ob sie einer bestimmten Gesetzmäßigkeit folgt.

Ein statistisches Gesetz, das die Distribution von Typen zu fassen sucht, ist das „Zipf'sche Gesetz“. Dieses Gesetz, das bereits 1935 von Zipf aufgestellt wurde, wird von Levshina (2015:64) folgendermaßen formuliert:

"According to this law, the corpus frequency of any word is inversely proportional to its rank in the frequency list. In other words, the first word on the frequency list is twice as frequent as the second most frequent word, etc. As a result, a corpus of natural language normally contains very few words with very high frequencies, which account for the larger part of the corpus, and very many words with low frequencies. This is why the majority of words that one can find in a corpus occur there only once. They are called hapax legomena (hapax legomenon in the singular)." 


\begin{tabular}{|c|c|c|c|c|c|c|c|}
\hline \multicolumn{2}{|c|}{ \# A tibble: $6,168 \times 2$} & \multirow{2}{*}{$\begin{array}{l}25 \text { aus } \\
26 \text { sich }\end{array}$} & \multirow{2}{*}{$\begin{array}{l}193 \\
188\end{array}$} & \multirow{2}{*}{$\begin{array}{l}50 \text { so } \\
51 \text { sagte }\end{array}$} & \multirow{2}{*}{$\frac{91}{89}$} & \multicolumn{2}{|c|}{77 wurde $\quad 55$} \\
\hline word & $\mathbf{n}$ & & & & & $78 \mathrm{ihr}$ & 54 \\
\hline$<\operatorname{chr}>$ & $<$ int $>$ & 27 eine & 184 & 52 nichts & 86 & 79 sei & 54 \\
\hline $1 \mathrm{ich}$ & 1038 & $28 \mathrm{als}$ & 170 & 53 zum & 86 & $80 \mathrm{da}$ & 52 \\
\hline 2 die & 982 & 29 großvater & 164 & $54 \mathrm{mehr}$ & 85 & 81 dieser & 50 \\
\hline 3 und & 802 & 30 wir & 164 & 55 meinem & 85 & 82 keine & 49 \\
\hline 4 der & 785 & 31 wie & 162 & 56 meiner & 80 & 83 sogenan & ten 48 \\
\hline 5 in & 713 & 32 noch & 161 & 57 sein & 79 & 84 uns & 47 \\
\hline 6 war & 583 & 33 nur & 151 & 58 hatten & 78 & 85 jetzt & 45 \\
\hline 7 das & 387 & 34 aber & 149 & 59 um & 78 & 86 ging & 44 \\
\hline 8 auf & 379 & 35 nach & 141 & 60 einer & 77 & 87 zwei & 44 \\
\hline 9 den & 369 & $36 \mathrm{im}$ & 138 & $61 \mathrm{man}$ & 77 & 88 mußte & 41 \\
\hline 10 nicht & 360 & $37 \mathrm{da} \beta$ & 137 & 62 vor & 76 & 89 schule & 41 \\
\hline $11 \mathrm{sie}$ & 349 & 38 waren & 133 & 63 oder & 71 & 90 vater & 41 \\
\hline 12 hatte & 348 & 39 einem & 125 & 64 gewesen & 70 & 91 einmal & 38 \\
\hline $13 \mathrm{zu}$ & 320 & 40 auch & 124 & 65 über & 70 & 92 großmut & er 38 \\
\hline $14 \mathrm{dem}$ & 313 & 41 immer & 118 & 66 alle & 69 & $93 \mathrm{ihm}$ & 38 \\
\hline $15 \mathrm{er}$ & 295 & 42 einen & 112 & 67 wieder & 68 & $94 \mathrm{ihn}$ & 38 \\
\hline 16 von & 294 & 43 für & 110 & 68 bei & 65 & 95 ihre & 38 \\
\hline 17 es & 289 & 44 mutter & 106 & 69 alles & 60 & 96 kein & 38 \\
\hline $18 \mathrm{mit}$ & 288 & 45 was & 103 & 70 habe & 60 & 97 meines & 38 \\
\hline 19 mich & 282 & 46 ist & 97 & 71 durch & 59 & 98 seine & 38 \\
\hline 20 ein & 264 & 47 wenn & 97 & 72 zeit & 59 & $99 \mathrm{du}$ & 37 \\
\hline 21 mein & 214 & 46 ist & 97 & 73 meinen & 58 & 100 haben & 37 \\
\hline 22 an & 209 & 47 wenn & 97 & 74 am & 57 & $\#$... with 6 & 68 more rows \\
\hline 23 meine & 209 & $48 \mathrm{des}$ & 92 & 75 diese & 57 & & \\
\hline $24 \mathrm{mir}$ & 205 & 49 schon & 92 & 76 bis & 55 & & \\
\hline
\end{tabular}

Tabelle (3)

Wenn wir nun die Verteilung der Gesamtheit der Typen des Romans von dem in Tabelle (3) nur ein Teil aufgelistet ist, in eine Grafik (2) unter dem Aspekt der Voraussagen des Zipf'schen Gesetzes überführen und betrachten, dann erkennen wir, dass die Verteilung der "types" grosso modo dem Gesetz entspricht. 


\section{Zipf-Verteilung}

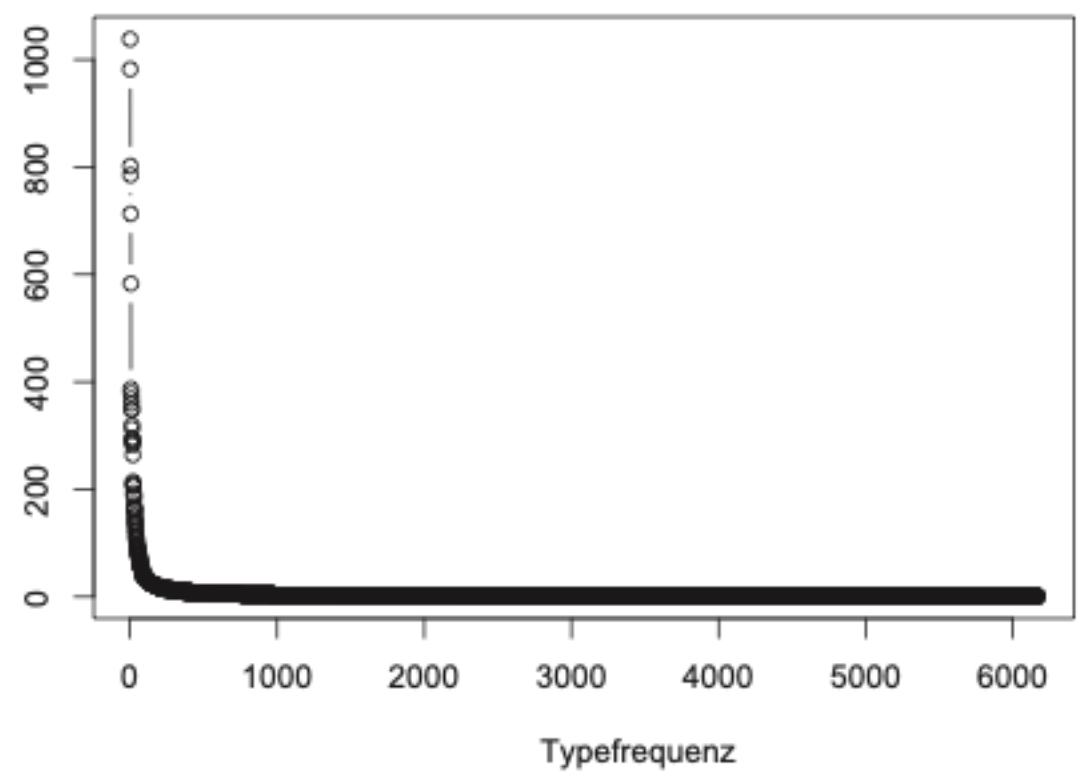

Grafik (2): Zipf'sche Verteilung der Types im Roman „Ein Kind“

Wir erkennen, dass das Korpus mehr als 6.000 (genau: 6.168) Typen hat und dass das „höchste“ Element auf der y-Achse eine Frequenz von mehr als 1.000 (genau:1038) aufweist, und so das Personalpronomen ,ich“ sein muss. Die dicke schwarze Linie parallel zur x-Ache zeigt das Resultat der grafische Konglomeration der Großteil der Typen, die nur eine niedrige Frequenz aufweist, und so dem Zipf'schen Gesetz entspricht. Im Detail bedeutet dies, dass es 5.538 Tokens gibt, welche 1-5 Mal im Text verwendet wurden. Die Details dazu ergeben sich aus den Tibbles von Tabelle (4), dessen Ergebnisse in Tabelle (5) zusammengefasst sind. Daraus ist zu entnehmen, dass beispielsweise „Abenteuer“ 2 Mal, „Anarchisten“ 3 Mal, „Bauern“ 4 Mal, und schließlich „Balkon“ 5 Mal im Text vorkommen.

Aus Tabelle (4) unten, und dann zusammengefasst in Tabelle (5), entnehmen wir, dass wir 3940 hapax legomena gefunden haben, also Wörter die jeweils nur 1 Mal im Roman vorkommen. Dazu gibt es im Roman 856 Wörter, die 2 Mal vorkommen, 399 Wörter die 3 Mal vorkommen und 214 Token, die 214 Mal vorkommen, und schlussendlich, 126 Wortformen, die jeweils 5 Mal vorkommen. Die Aufstellung zeigt aber auch, dass die Anzahl der Wörter, die öfters vorkommen, bereits in dieser beschränkten Auflistung abnimmt, so dass die Wörter, die jeweils 5 Mal im Text vorkommen, nur mehr einen Bruchteil der Anzahl der hapax legomena ausmachen. 


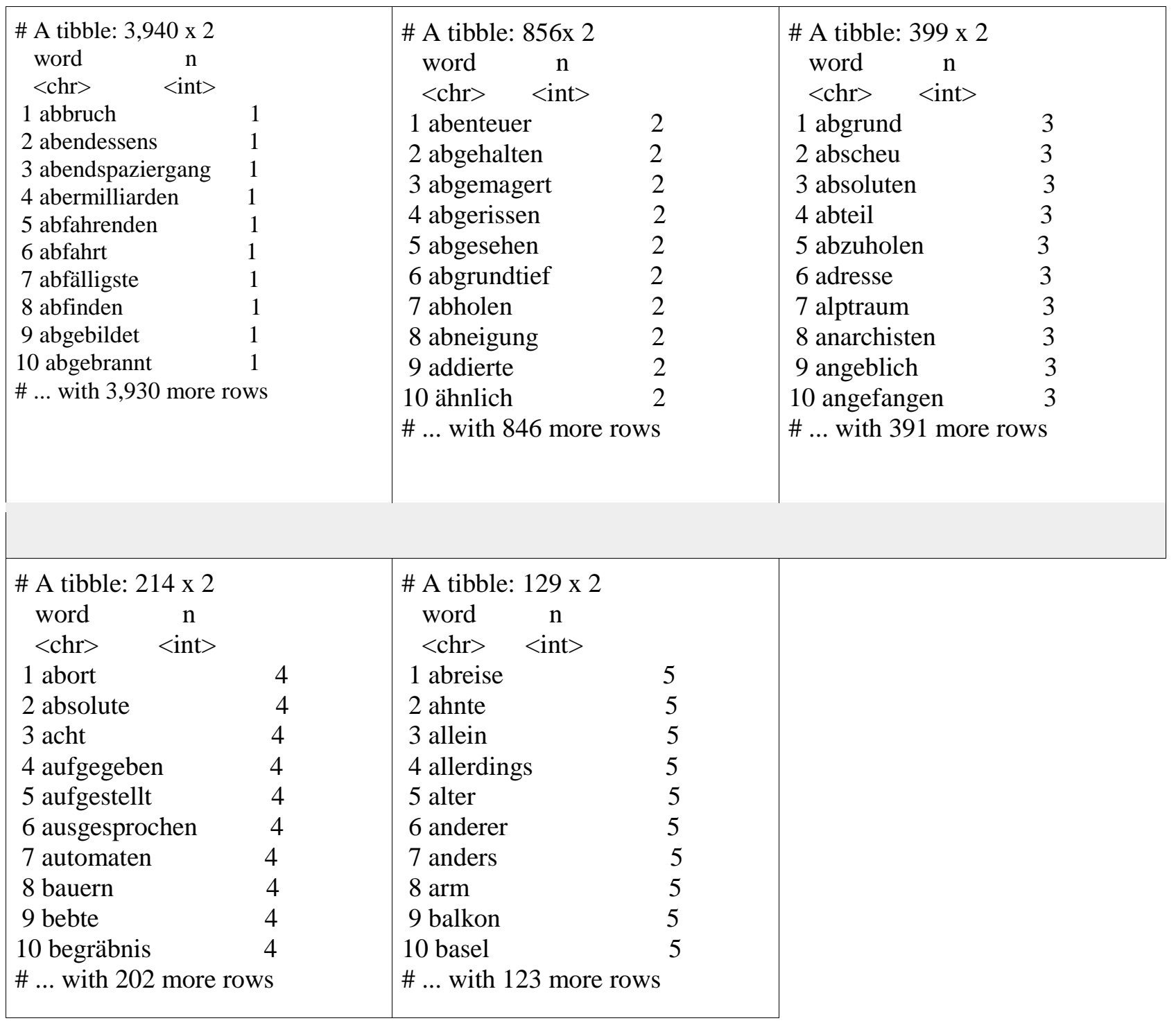

Tabelle (4): Beispiele für Typenfrequenz von (1-5)

\begin{tabular}{|r|r|}
\hline Anzahl (n) & Vorkommen (Type) \\
3940 & 1 \\
856 & 2 \\
399 & 3 \\
214 & 4 \\
129 & \\
5538 & Spannweite (1-5) \\
\hline
\end{tabular}

Tabelle (5)
Diese Quantitätsverteilungen zeigen auf, dass $89,78 \%$ der Typen in Bernhards Text 1-5 Mal vorkommen, während nur 10,22\% der Typen mehr als 5 Mal vorkommen. Wir erhalten dadurch eine Frequenzrelation von 1598:3940. Dies bedeutet wiederum, dass die Anzahl der hapax legomena $2 \mathrm{Mal}$ so hoch ist wie die Summe der Typen, die jeweils 2-5 Mal vorkommen. Wie jedoch schon mit Grafik (2) der Zipf'schen Verteilung der Types gezeigt wurde, können wir schlussfolgernd annehmen, dass die quantitativen Verhältnisse der Wortverwendung von Thomas Bernhard grosso modo den Voraussagen des Zipf'schen Gesetzes entsprechen. 
Diese erste Annäherung an das Phänomen der "lexical richness" im Roman von Thomas Bernhard ist jedoch ohne Vergleich mit anderen Texten aus dem literarischen Genre nur bedingt nützlich, da es sich um ein Singularergebnis handelt, das nur mit Vorbehalt generalisiert werden soll. Weitere, diesbezügliche Studien sollten zeigen, ob quantitative Charakteristika im Bereich der Literatur im Vergleich zu „Gebrauchstexten“ von einem solchen Ausmaß sind, dass wir von einer Sonderstellung des Texttyps „Literatur“ sprechen können.

Wir haben oben zeigen können, dass die Anzahl der nominalen Referenzen zur engeren Verwandtschaft von Thomas Bernhard im Vergleich zur Verwendung anderer Nomina als vergleichsweise hochfrequent bezeichnet werden kann. Da die absoluten Frequenzen aber die Frage nicht beantworten, ob die wichtigsten Protagonisten im Roman „Ein Kind“ immer in gleichen oder ähnlichen Abständen im Text erwähnt werden, soll hier dieser Frage mit einer Distributionsanalyse nachgegangen werden. Das Ergebnis dieser Analyse zeigt Grafik (3), die als Datenbasis die Referenzen von seinem „Großvater“ (164 Erwähnungen, Rang 29), seiner „Mutter“ (106 Erwähnungen, Rang 44), von seinem „Vater“ (41 Erwähnungen, Rang 90), und seiner „Großmutter“ (38 Erwähnungen, Rang 92) hat. Wie aber die f-Dispersiongrafik (3), welche jedes Vorkommen eines Protagonisten mit einer vertikalen Linie darstellt, und die somit als Zeitlinie des Romans im Sinne einer „Ereignislinie“ zwischen „Romanbeginn“ und des „Romanende“ interpretiert werden kann, zeigt, tritt nur der Hauptprotagonist (,ich“), so regelmäßig im Text auf, dass dieses Auftreten oft zu einer Schwärzung der Ereignislinie führt. Die anderen Personen, weisen dagegen Referenzlücken auf, die durch leere Flächen in der Grafik dargestellt werden. Mit Abstand hat „der Großvater“, der ja auch die meisten Nennungen erfährt, und sicherlich als die primäre Bezugsperson von Thomas Bernhard bezeichnet werden kann, im Roman nicht nur die meisten Nennungen, sondern auch ein fast durchgehend konstantes Auftreten im Roman. Die anderen Protagonisten erfahren in Teilen des Romans eine geringere, oder auch gar keine Erwähnung. Ihr Auftreten zeigt unregelmäßige Abstände auf. Diese Analyse zeigt also, dass „Textfrequenz" nicht unbedingt bedeutet, dass die Token in allen Teilen eines Textes in gleichmäßigen Abständen auftreten müssen und eine Streuung unregelmäßig sein kann, was wiederum bedeutet, dass die Protagonisten des Romans „Ein Kind“ in unregelmäßigen Abständen in die Handlung eingebunden sind und in welchen Textteilen diese stattfindet. ${ }^{9}$

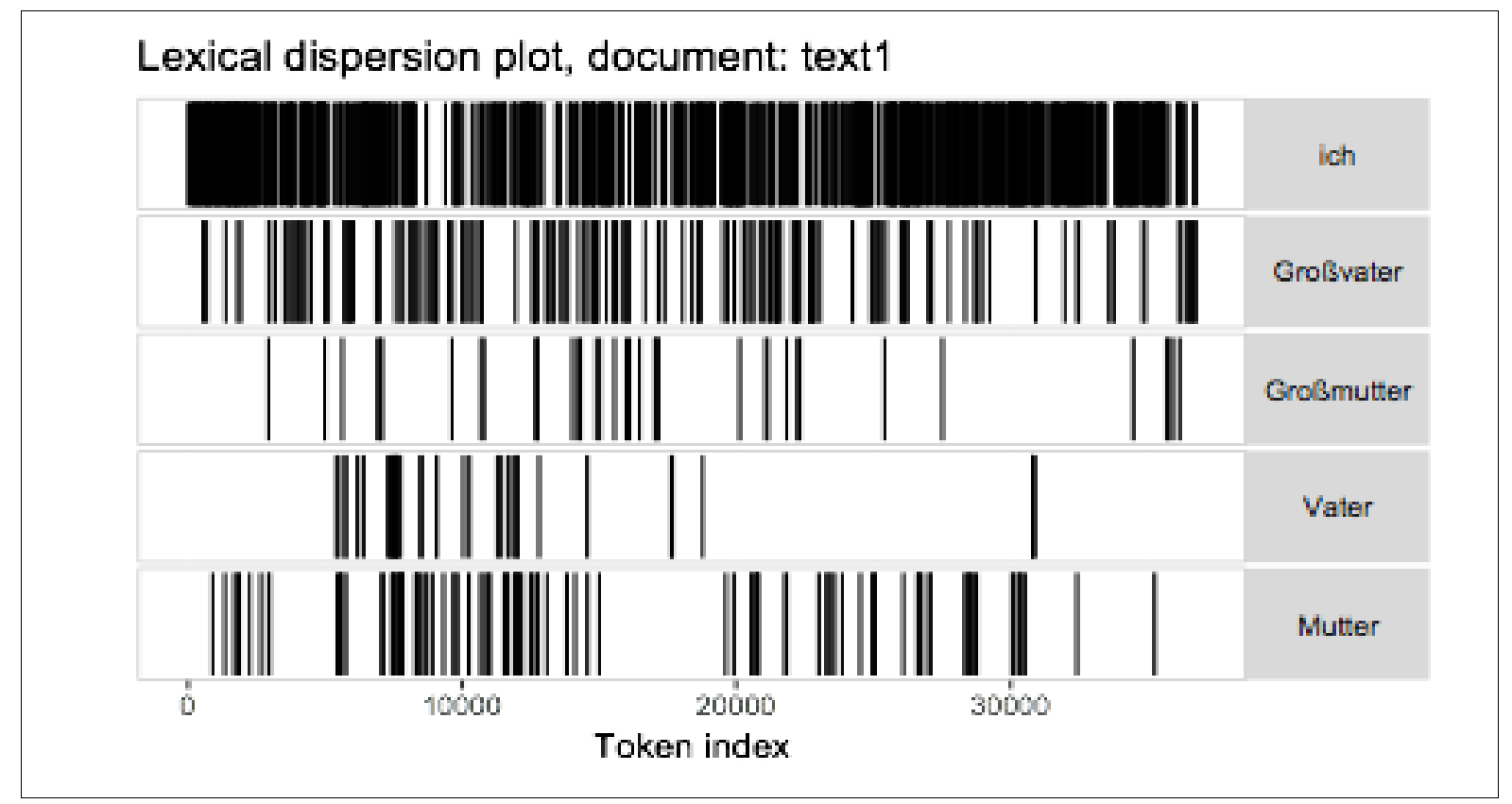

Grafik (3): Dispersionsplot der Protagonisten ,,ich“, „, Großvater“, „, Großmutter“, ,, Vater “ und „, Mutter“ im Roman „Ein Kind"

\footnotetext{
${ }^{9}$ Es ist zu beachten, dass für die Grafik nur das Auftreten der angeführten Unigramme verwendet wurde. Dies bedeutet u. a., dass eine Buchstabenfolge wie „Großvaters“, oder „Vaters“ unbeachtet blieb. Die Einbeziehung solcher Wortformen hätte das Ergebnis aber nur sehr geringfügig beeinflusst.
} 


\section{$3 \quad$ Analyse von Bigrammen und darüber hinaus}

In den vorigen Abschnitten wurden die Frequenzen von Einzeltokens und Einzeltypes, also Unigrammen, angeführt. Im Folgenden wird - im Unterschied dazu - der Frage nachgegangen, ob wir im Text auch Mehrfachsequenzen von Wortformen finden und welche Charakteristik und Quantität diese aufweisen. Die allgemeinen Funktionen des Programms R für diese Analyse sind "bigram", "sort" und "count" sowie weitere Spezifizierungen derselben.

Das Resultat der einfachen Bigramm-Analyse zeigt Tabelle (6), welche wie auch Tabelle (7) Kopien des Programmoutputs des Analysetools R sind:

\author{
\# A tibble: $29,028 \times 2$ \\ line bigram \\ $<$ int $><$ chr $>$ \\ 11 im alter \\ 21 alter von \\ 31 von acht \\ $4 \quad 1$ acht jahren \\ 51 jahren trat \\ 61 trat ich \\ 71 ich auf \\ 81 auf dem \\ 91 dem alten \\ 101 alten steyr \\ \# ... with 29,018 more rows
}

\begin{tabular}{lr} 
\# A tibble: & $20,735 \times 2$ \\
bigram & $\mathrm{n}$ \\
<chr> & \multicolumn{2}{c}{ <int> } \\
1 in der & 125 \\
2 mein großvater & 92 \\
3 in die & 81 \\
4 auf dem & 65 \\
5 ich war & 64 \\
6 ich hatte & 60 \\
7 es war & 57 \\
8 meine mutter & 55 \\
9 auf die & 49 \\
10 hatte ich & 49 \\
\# ... with 20,725 more rows
\end{tabular}

Tabelle (6)

Tabelle (7)

Wir sehen, dass das Statistikprogramm ein Tibble mit 29,028 Bigramme ausgibt. Diese Bigramme sind das Resultat der binären Kombination von Einzeltokens, welche sequentiell als ab, bc, cd, usw. kombiniert, gezählt und ausgegeben werden. Konkret bedeutet dies, dass die Wortfolge des Romananfangs, also „Im Alter von acht Jahren“ wie folgt aufgelistet und gezählt wird: ,Im Alter“; ,Alter von“; ,von acht‘; und ,acht Jahren“. Da die Bigramme dann „zeilenweise“ gezählt werden, haben wir folglich 4 verschiedene Bigramme. Wenn das Auftreten der Bigramme von einzelnen Wortformen aber summiert wird, dann erhalten ein Tibble mit 20.735 Zeilen, bzw. Bigramm-Typen. Wie wir sehen, werden die Bigramm-Typen auch nach ihrem Rang, und im Rang in alphabetischer Reihenfolge geordnet und aufgelistet. Durch eine solche Analyse erhalten wir beispielsweise die Information, dass das Bigramm ,,in der“ im Text 125 Mal vorkommt und zugleich Rang 1 unter den Bigramm-Typen des Korpus einnimmt. In Bezug auf die im Roman erwähnten engeren Verwandten von Thomas Bernhard bedeutet dies nun, dass das Bigramm „mein Großvater“ im Roman 92 Mal mit Rang 2 aufscheint. Der Bigramm-Typ „meine Mutter“ erscheint 55 Mal mit Rang 8, „meine Großmutter“ 24 Mal mit Rang 38 und „mein Vater“ 5 Mal mit Rang 460. Da das Deutsche jedoch infolge von Kasus und Geschlecht unterschiedliche Wortformen verlangt, müssen für eine adäquate Zählung der Gesamtanzahl der Referenzen von Thomas Bernhards enger Verwandtschaft auch Kasus und Genus der besagten Bigramme berücksichtigen. Diese Sequenzen müssen mit der hier verwendeten Methode getrennt eruiert und summiert werden, um ein getreues, quantitatives Abbild 
„der Präsenz“ dieser Protagonisten in dem Werk „Ein Kind“ zu geben. Tabelle (8) berücksichtigt diese Aspekte der deutschen Sprache, da darin die Nennungen nach Genus und Kasus aufschlüsselt sind.

Tabelle (8) zeigt, dass die Kasusvarianten von „mein/meines/meinem/meinen Großvater“ eine Gesamtfrequenz von 147 Instanzen aufweisen. Für „meine/meiner Mutter“ zählte das Programm insgesamt 82, für „meine/meiner Großmutter“ 30, und letztlich 14 Instanzen für „mein/meines/meinem/meinen Vater“. Dies bedeutet, dass von 349 Nennungen der Unigramme „Großvater“, „Mutter“, „Großmutter“ und „Vater“ ein Großteil (d. s. 273, und somit 78,22\%) mit dem Possessivpronomen vorkommen. Der Rest kommt mit definitem Artikel vor. Auch ist ersichtlich, dass sich die Opposition zwischen ,Großvater" ohne Possessivpronomen und mit Possessivpronomen eine Relation von 164:147, ergibt, die zwischen ,Mutter ${ }^{6}$ mit und ohne Possessivpronomen eine von 106:82, zwischen ,Großmutter ' ohne Possessivpronomen und mit Possessivpronomen eine von 38:30 und zwischen ,Vater' ohne Possessivpronomen und mit Possessivpronomen zwischen 41:14 ergibt. Die größte Diskrepanz ergibt sich also bei „Vater“, der nur 14 Mal mit „mein Vater/meines Vaters/meinem Vater/meinen Vater“ erwähnt wird. Dies kann mit dem nicht harmonischen Verhältnis zwischen dem „unbekannten“ Vater und seinem Sohn zusammenhängen. Die durch die Analyse der Bigramme gefundenen Quantitäten zeigen somit, dass Thomas Bernhard eine Wortwahl mit dem Possessivpronomen bevorzugt. ${ }^{10}$

\begin{tabular}{|c|c|c|c|c|c|c|c|}
\hline Bigramm 1 & $\mathrm{n}$ & Bigramm 2 & $\mathrm{n}$ & Bigramm 3 & $\mathrm{n}$ & Bigramm 4 & $\mathrm{n}$ \\
\hline $\begin{array}{l}\text { mein } \\
\text { großvater }\end{array}$ & 92 & $\begin{array}{l}\text { meine } \\
\text { mutter }\end{array}$ & 55 & $\begin{array}{l}\text { meine } \\
\text { großmutter }\end{array}$ & 24 & $\begin{array}{l}\text { mein } \\
\text { vater }\end{array}$ & 5 \\
\hline meines großvaters & 15 & meiner mutter & 27 & meiner großmutter & 6 & meines vaters & 1 \\
\hline $\begin{array}{l}\text { meinem } \\
\text { großvater }\end{array}$ & 28 & & & & & meinem vater & 3 \\
\hline $\begin{array}{l}\text { meinen } \\
\text { großvater }\end{array}$ & 12 & & & & & $\begin{array}{l}\text { meinen } \\
\text { Vater }\end{array}$ & 5 \\
\hline Gesamt & 147 & & 82 & & 30 & & 14 \\
\hline
\end{tabular}

Tabelle (8): Gesamtzahl der Bigramme von „,mein/es/em/en Großvater“, „,meine/r Mutter", „,meine/r Großmutter" und „,mein/es/em/en Vater"

Wie schon ein Mal angeführt, nimmt das Bigramm ,in der“ mit 125 Nennungen im Roman den 1. Rang unter diesen zweigliedrigen Wortsequenzen ein. Wie die Analyse weiters zeigt, weisen die Bigramme nicht wirklich überraschend eine abfallende Frequenzhierarchie auf. Sie beginnen mit 125 Instanzen für ,in der“ und enden bei 17.885 Bigrammen in singulärer Verwendung. Letztere sind jedoch für die Fragestellung dieses Aufsatzes von geringerem Interesse, da sie nichts über die mehrmalige Verwendung von Lexemsequenzen aussagen. Im Vergleich dazu treten 27.980 Bigramme je 2 Mal auf. Um einen Einblick in die beschriebenen Tätigkeiten und Zustände von Thomas Bernhard zu geben, werden in Tabelle (9) 55 Bigramme aufgelistet, die jeweils 2 Mal vorkommen, mit „ich“ beginnen und Ränge wie 1979, 1980, 1981, etc. haben. Wir sehen hier auch eineindeutig die Auflistungsfunktion des Programms: Obwohl alle Sequenzen gleich oft vorkommen, erhalten sie hier einen unterschiedlichen Rang, weil auch die Alphabetisierung zur Rangzuordnung des jeweiligen

${ }^{10}$ Die Suche nach der Frequenz von morphologisch unterschiedlichen Wortformen kann auch mit RegexAusdrücken erfolgen und kann damit vielleicht etwas effizienter bewerkstelligt werden. 
Bigrammes als Kriterium herangezogen wird. ${ }^{11}$ Diese Zählweise bedingt somit, dass frequenzmäßig gleiches Auftreten von individuellen Lexemfolgen nicht unweigerlich ,gleicher Rang“ bedeutet.

\begin{tabular}{|c|c|c|c|}
\hline 1979 ich allein & 1993 ich eine & 2007 ich mehr & 2021 ich sicher \\
\hline 1980 ich als & 1994 ich er & 2008 ich meinem & 2022 ich sogar \\
\hline $1981 \mathrm{ich}$ am & 1995 ich fand & 2009 ich meinen & 2023 ich spielte \\
\hline 1982 ich angst & 1996 ich ganz & 2010 ich nach & 2024 ich steigerte \\
\hline $1983 \mathrm{ich}$ & 1997 ich getraute & $2011 \mathrm{ich}$ & 2025 ich stieg \\
\hline aufwachte & 1998 ich glaubte & naturgemäß & 2026 ich tat \\
\hline 1984 ich bald & 1999 ich heulte & 2012 ich nichts & 2027 ich trug \\
\hline 1985 ich begriff & 2000 ich höre & 2013 ich oder & 2028 ich unfähig \\
\hline 1986 ich beinahe & $2001 \mathrm{ich}$ ihr & 2014 ich sagte & $2029 \mathrm{ich}$ \\
\hline 1987 ich bemühte & $2002 \mathrm{ich} \mathrm{im}$ & 2015 ich salzburg & verdiente \\
\hline 1988 ich brachte & 2003 ich jemals & 2016 ich scheiterte & 2030 ich wenn \\
\hline 1989 ich bringe & 2004 ich kauerte & 2017 ich schlief & 2031 ich wieder \\
\hline 1990 ich denke & 2005 ich kniete & 2018 ich schrieb & 2032 ich wurde \\
\hline 1991 ich denken & 2006 ich lernte & 2019 ich sehe & 2033 ich zog \\
\hline 1992 ich doch & & 2020 ich sehr & \\
\hline
\end{tabular}

Tabelle (9) Bigramme mit , ich “ mit zweimaligem Vorkommen

Wenn wir im folgenden Abschnitt der Frage nachgehen, ob sich im Roman „Ein Kind“ noch mehr Sequenzen im Sinne von Trigrammen, Quagrammen und Quingrammen befinden, dann kann diese Frage uneingeschränkt bejaht werden.

Die Analyse der Trigramme zeigt, dass die Gesamtsumme dieser Sequenzen 25.301 beträgt. Sie weisen eine Typenstreuung von 12 Instanzen (Maximum) bis zum Minimum von 1 Instanz auf und unterscheiden sich daher im Vorkommen sehr von den Bigramm-Typen des vorigen Abschnittes. Die Typen mit einer Frequenz von 12 bis 6 sind zur Illustration in Tabelle (10) aufgelistet.

Wir sehen hier, dass Bernhards Großvater auch hier wieder mit 3 Typen von Trigrammen vertreten ist. Das Trigramm ,sagte mein Großvater“ nimmt Rang 1 ein und kommt im Text $12 \mathrm{Mal}$ vor, das Trigramm „mein Großvater hatte” nimmt Rang 3 ein und wurde im Roman 10 Mal verwendet. Das Trigramm „von meinem Großvater“ wurde 6 Mal verwendet und nimmt dadurch den Rang 19 unter den Trigrammen ein. Das Programm weist weiters aus, dass 751 Trigamm-Typen mehr als 1 Mal vorkommen. Dies bedeutet wiederum, dass 24.550 Trigramme nur 1 Mal vorkommen und 2,97\%, also fast 3 Prozent ausmachen. Im Vergleich dazu hat der Text insgesamt 20.735 BigrammTypen und 2854, die öfters als 1 Mal auftreten. Das bedeutet, dass 13,76\% der Bigramm-Typen keine Unikate sind.

\footnotetext{
${ }^{11}$ Die Auflistung dieser Bigramm-Types in Tabelle (9) bedeutet nicht, dass solche Lexemsequenzen nicht öfters vorkommen. So kommt beispielsweise ,ich war“ 64 Mal vor, ,ich hatte“ 60 Mal, ,ich liebte“ 11 Mal und ,ich weiß" 8 Mal. Diese Gruppe von Tabelle (9) wurde gewählt, weil sie die größte unter den Bigramm-Typen darstellt, die öfters als 1 Mal im Roman vorkommt.
} 


\begin{tabular}{|lr|}
\hline > trigrams.ek\%>\% & \\
\# A tibble: $25,301 \times 2$ & \\
bigram & \multicolumn{2}{|c|}{} \\
<chr> & \\
1 sagte mein großvater & 12 \\
2 in die schule & 11 \\
3 mein großvater hatte & 10 \\
4 in der nähe & 9 \\
5 ich war nicht & 8 \\
6 ich hatte einen & 7 \\
7 ich in die & 7 \\
8 die meiste zeit & 6 \\
9 die poschinger elli & 6 \\
10 es sich um & 6 \\
11 es war ein & 6 \\
12 es war mir & 6 \\
13 ich liebte die & 6 \\
14 im ersten stock & 6 \\
15 in der schule & 6 \\
16 in der wernhardtstraße & 6 \\
17 in dieser zeit & 6 \\
18 nichts anderes als & 6 \\
19 von meinem großvater & 6 \\
\hline
\end{tabular}

Tabelle (10): Top-Trigramme

Zum Vergleich mit den Bigramm-Typen, die an erster Stelle der Sequenz das Pronomen „ich“ aufweisen, sind in der folgenden Tabelle (11) Trigramme aufgeführt, die ebenso 2 Mal vorkommen und bei denen in der ersten Position der Sequenz auch das Pronomen ,ich“ vorkommt. ${ }^{12}$ Es handelt sich hier nur mehr um 46 Trigramm-Typen:

\footnotetext{
${ }^{12}$ Um zielführend über quantitative Aspekte von Bigrammen mit dem Ich-Erzähler als Agens wie in ,ich verdiente“, „ich spielte“, „ich denke“, „ich heulte“, „ich kniete“, etc. zu erhalten, bedarf es einer TaggingAnalyse, welche die Wortarten spezifiziert, da ansonsten mit der hier verwendeten Methode auch Sequenzen wie „,ich allein“, „ich oder“, „,ich ihr“, etc. im Output gefunden werden. Eine andere Möglichkeit bestünde auch darin, jede unnötige Sequenz „manuell“ auszufiltern. Von diesen Möglichkeiten wird hier aber kein Gebrauch gemacht, da die Behandlung von semantisch-pragmatischen Aspekten des Romans außerhalb des Bereichs dieses Aufsatzes liegt.
} 


\begin{tabular}{|c|c|c|c|c|c|}
\hline 412 ich ahnte daß & 2 & 428 ich hatte von & 2 & 444 ich nicht ich & 2 \\
\hline 413 ich an der & 2 & 429 ich hatte zwei & 2 & 445 ich nicht mehr & 2 \\
\hline 414 ich aufeinmal war & 2 & 430 ich ich war & 2 & 446 ich noch heute & 2 \\
\hline 415 ich aufwachte war & 2 & 431 ich kannte die & 2 & 447 ich schon in & 2 \\
\hline 416 ich aus dem & 2 & 432 ich liebte meine & 2 & 448 ich sei ein & 2 \\
\hline 417 ich bemühte mich & 2 & 433 ich lief mit & 2 & 449 ich stand auf & 2 \\
\hline 418 ich bin ein & 2 & 434 ich ließ mich & 2 & 450 ich steigerte mich & 2 \\
\hline 419 ich bis dahin & 2 & 435 ich mich an & 2 & 451 ich über alles & 2 \\
\hline 420 ich das wort & 2 & 436 ich mich ganz & 2 & 452 ich war so & 2 \\
\hline 421 ich dazu kam & 2 & 437 ich mich nach & 2 & 453 ich war stolz & 2 \\
\hline 422 ich getraute mich & 2 & 438 ich mich nicht & 2 & 454 ich weiß nicht & 2 \\
\hline 423 ich hatte auf & 2 & 439 ich mich noch & 2 & 455 ich will ich & 2 \\
\hline 424 ich hatte in & 2 & 440 ich mir eine & 2 & 456 ich wußte gar & 2 \\
\hline 425 ich hatte nicht & 2 & 441 ich mit der & 2 & 457 ich wußte wofür & 2 \\
\hline 426 ich hatte noch & 2 & 442 ich mit ihr & 2 & & \\
\hline 427 ich hatte schon & 2 & 443 ich nach dem & 2 & & \\
\hline
\end{tabular}

Tabelle (11): Trigramme

Wir können auf der Grundlage der Information aus der bisherigen Analyse zweifellos behaupten, dass Bernhard Sequenzen von Lexemen verwendet, die sich wiederholen. Dies zeigte sich durch die Analyse und Quantifizierung von Bigrammen und Trigrammen, die mehr als 1 Mal im Roman verwendet werden. Es stellt sich nun die Frage, ob auch Quingramme und Quagramme als weitere Belege für Wiederholungssequenzen liefern. Dies ist in der Tat der Fall.

Wie zu erwarten, reduziert sich die Gesamtzahl der Quagramme auf 23.614 Typen. Die TopFrequenz nimmt dabei die Sequenz ,in der Nähe des“ mit 5 Verwendungen ein. Darauf folgt „Ich erinnere mich daß“ und „Steyr Waffenrad meines Vormundes“ mit jeweils 4 Frequenzen. Dazu gibt es 9 „Zeilen“, die die dreimalige Verwendungen von Wortfolgen des Typs „Quagram“ belegen. Die Anzahl von Quagrammen, welche zweifach vorkommen, beträgt 101. Von der bereits genannten Zahl von 23.615 Quagrammen kommen 113 öfters als 1 Mal vor. Dies sind 0,48\%, also ca. 0,5 Prozent. Wir haben jetzt auch nur mehr 5 Quagramme, die mit dem Personalpronomen „ich“ beginnen. Diese Zahlen zeigen somit eine drastische Reduktion der Anzahl der Lexemsequenzen an, die wiederholt im Text auftreten. Es stellt sich demnach in der Folge auch die Frage, ob es überhaupt Quingramme gibt?

Die Analyse der Quingramme zeigt, dass das Programm 21.076 Quingramme finden und auflisten konnte. Wir sehen aber auch, dass nur 24 Quingramme öfters als 1 Mal vorkommen und die Topfrequenz von 3 Verwendungen die Phrase von Bernhards Mutter ist, die „Du hast mir noch gefehlt“, lautet. Abgesehen von der Phrase „Ich erinnere mich daß ich“, welche auch 3 Mal vorkommt, 
gibt es dann nur noch 12 Belege von Sequenzen, die jeweils 2 Verwendungen anzeigen. Auch die Zahl der Sequenzen, welche mit dem Personalpronomen „ich“ beginnen, ist wie Tabelle (12) zeigt, drastisch reduziert und besteht jetzt aus 3 Einträgen:

\begin{tabular}{|ll|}
\hline 13 ich in die schule ging & 2 \\
14 ich war nicht so dumm & 2 \\
15 ich war stolz auf sie & 2 \\
\hline
\end{tabular}

Tabelle (12): Quagramme

Kurz soll noch erwähnt werden, dass es auch Sextgramme im Roman gibt. Von den 18.458 gezählten Sextgrammen sind jedoch nur 4 Typen mit jeweils 2 Wiederholungen gefunden worden. Diese Gruppe bringt somit keine neuen Erkenntnisse in Bezug auf die frequenzmäßige Verschiedenheit von Tokenwiederholungen, so dass deren Analyse nicht weiterverfolgt wird.

\section{$4 \quad$ Zusammenfassung}

Wir erkennen durch die hier angestellten Frequenzuntersuchungen, dass die Anzahl der Phrasenwiederholungen mit der Länge der untersuchten Sequenzen - wie sie in Trigramme, Quagramme und Quingramme wurden, ziemlich drastisch abnimmt. Die Frage, ob es sich diesbezüglich um das Resultat intuitiver Sprachverwendung handelt, oder ob es sich um eine bewusste, schriftstellerische Konstruktion Sprachsequenzen in Texten handelt, kann mit der hier verwendeten Methode nicht beantwortet werden, so dass wir zu einer anderen Fragestellung übergehen müssen. Wenn wir aber nun idealiter annehmen, dass ein guter Schriftsteller absolut keine Lexeme und auch keine Lexemsequenzen verwendet, die mehrfach vorkommen, und sich dessen auch bewusst ist, dann können wir aus der hier vorgestellten Analyse schließen, dass Thomas Bernhard zwar interessante und spannende Handlungen produzierte, die viele Leser immer noch begeistern, dass aber der Roman „Ein Kind“ nur geringe linguistische Kreativität in Bezug auf Lexik- und Phrasenverwendung aufweist, da wir ziemlich viele Bigramme, Trigramme und vielleicht auch Quagramme finden, die sich wiederholen. Da die hier benützte Datenbasis aber nur aus einem Text besteht und somit kein Vergleich mit anderen Werken von Bernhard, oder auch Schriftstellern angestellt wurde, sind die hier angestellten Überlegungen nur als eine Diskussionsgrundlage zu verstehen, die durch Anwendung von konventionellen Analysen der Korpuslinguistik erreicht wurde. Die damit erzielten Resultate müssen vielleicht gänzlich verworfen, bestimmt aber relativiert werden.

\section{Bibliografie}

Baayen, Harald R. 2008. Analyzing Linguistic Data: A Practical Introduction to Statistics Using R. Cambridge: Cambridge University Press.

Desagulier, Guillaume. 2017. Corpus Linguistics and Statistics with R: Introduction to Quantitative Methods in Linguistics. Heidelberg: Springer Verlag.

Gries, Stefan Th. 2015. "50-something years of work on collocations. What is or should be next..." In Sebastian Hoffmann, Bettina Fischer-Starcke, \& Andrea Sand (eds.), Current issues in phraseology, 135-164. Amsterdam \& Philadelphia: John Benjamins.

Gries, Stefan Th. 20172. Quantitative Corpus Linguistics with R: A Practical Introduction. New York: Routledge.

Huber, Martin \& Manfred Mittermayer (Hg.). 2004. Thomas Bernhard: Werke in 22 Bänden - Band 10: Die Autobiographie. Frankfurt am Main: Suhrkamp Verlag.

Jokers, Matthew L. 2014. Text Analysis with R for Students of Literature. Heidelberg: Springer Verlag. 
Levshina, Natalia 2015. How to do Linguistics with R: Data exploration and statistical analysis. Amsterdam: John Benjamins Publishing Company.

Perkuhn, Rainer, Holger Deibel, \& Marc Kupietz. 2012. Korpuslinguistik. München: Wilhelm Fink.

Silge, Julia \& David Robinson. 2017. Text Mining with R: A Tidy Approach. Beijing: O’Reilly.

Stopwords in 40 Sprachen: https://www.ranks.nl/stopwords

Wickham, Hadley \& Garrett Grolemund. 2017. R for Data Science: Import, Tidy, Transform, Visualize, and Model Data. Beijing: O’Reilly.

Wickham, Hadley. 2017. Easily Install and Load the 'Tidyverse'.

https://CRAN.R-project.org/package=tidyverse

Wickham, Hadley, and Romain Francois. 2016. ‘dplyr: A Grammar of Data Manipulation.’ https://cran.rproject.org/package=Tidyr

Zipf, G. K. 1935. The Psycho-Biology of Language. Cambridge, MA.: MIT Press. 